\title{
Germanica
}

\section{Krise in Serie. Der Fall der Weimarer Republik in den Kriminalromanen Volker Kutschers}

Crise en série. La chute de la République de Weimar dans les romans policiers de Volker Kutscher

Crisis in Series. Investigations in the Weimar Republic in Volker Kutscher's crime novels

\section{Sandra Beck}

\section{OpenEdition}

\section{Journals}

Édition électronique

URL : http://journals.openedition.org/germanica/3193

DOI : 10.4000/germanica.3193

ISSN : 2107-0784

\section{Éditeur}

Université de Lille

\section{Édition imprimée}

Date de publication : 30 septembre 2016

Pagination : 111-120

ISBN : 9782913857377

ISSN : 0984-2632

\section{Référence électronique}

Sandra Beck, « Krise in Serie. Der Fall der Weimarer Republik in den Kriminalromanen Volker Kutschers », Germanica [Online], 58 | 2016, Online erschienen am: 30 September 2018, abgerufen am 06 Oktober 2020. URL : http://journals.openedition.org/germanica/3193 ; DOI : https://doi.org/ 10.4000/germanica.3193 


\title{
Krise in Serie \\ Der Fall der Weimarer Republik in den Kriminalromanen Volker Kutschers
}

\author{
Sandra BECK \\ Universität Mannheim
}

It is seldom that liberty of any kind is lost all at once.

David Hume ${ }^{1}$

Die historische Forschung hat in ihrer Auseinandersetzung mit den Jahren 1918 bis 1933 eine dichte Krisenerzählung ausgearbeitet, deren Untergliederung in drei Erzählabschnitte den einschlägigen Studienhandbüchern ihre Struktur verleiht: Ausgehend von „Entstehung und Selbstbehauptung“ der Demokratie (1918-1923) über die „Phase der relativen Stabilisierung“ (1924-1929) bis hin zu „Auflösung und Zerstörung“ (1930-1933)2 zeichnet sie ein so stringentes wie kausallogisches Protokoll einer „Culture in Crisis and a Culture of Crisis“3, die sich mit dem Aufstieg des Dritten Reiches entscheidet. Diese historiographische Erzählung von einer Krisenzeit sieht sich dabei methodisch ebenso herausgefordert wie bestätigt von den zahlrei-

1. - Dieses Zitat aus Of the Liberty of the Press (1742) ist dem dritten Teil von Volker Kutschers Roman Die Akte Vaterland als Motto vorangestellt.

2. - Eberhard Kolb, Dirk Schumann, Die Weimarer Republik, München, Oldenbourg, ${ }^{82013 .}$

3. - Todd Herzog, Crime Stories. Criminalistic Fantasy and the Culture of Crisis in Weimar Germany, New York, Oxford, Berghahn Books, 2009, S. 1. 
chen zeitgenössischen Quellen, in denen „Krise ${ }^{6}$ als zentrales Moment gesellschaftlicher Selbstbeschreibung erscheint ${ }^{4}$. Angesichts dieser „metaphorische[n] Ausdehnung“5 des Krisen-Begriffs in der kritischen Wahrnehmung der Zeitgenossen und vor dem Hintergrund des Sinn- und Deutungszusammenhangs der Krise als historiographischem Metanarrativ ist man versucht, für die Weimarer Republik mitErnst Bloch lakonisch zu resümieren: „Gute Zeit also für Detektivisches schlechthin “6. Denn detektivisches Erzählen lässt sich beschreiben als narrative Experimentalanordnung, in derdieErzählung der Aufklärungsgeschichte beobachtbar macht, wie die Ermittlungsinstanzen im (epistemologischen) Kampf mit dem Verbrecher die durch den Mord aus den Fugen geratene Welt aus ihrem Zustand des Zweifels und des Chaos erlösen. Die narrative Doppelstruktur des Genres koppelt so die Simulation des Ausnahmezustandes stets an das Erlösungsversprechen des Erkennens - gleichsam wie in einem ,rückwärtslaufenden Film [...], der eigentlich das Zerspringen eines von einer Kugel getroffenen Spiegels zeigen sollte, statt dessen aber dem faszinierten Publikum vorführte, wie sich Splitter um Splitter wieder zu einer blanken Fläche fügten"7.

Die in Serie geschalteten und zur Verfilmung als Serie vorgemerkten historischen Kriminalromane Volker Kutschers ${ }^{8}$ zeichnen sich nun aus durch ein bedenkenswertes Spiel mit ,Krise ${ }^{6}$ als historiographischem Metanarrativ, als zeitgenössischer Diagnose und als Funktionsformel kriminalliterarischen Erzählens. Während Hardy Reich anlässlich der Veröffentlichung des ersten Gereon Rath-Romans vermerkte, der Autor plane nichts weniger als „den Untergang der Weimarer Republik im Medium des Kriminalromans darzustellen"9 , betont Kutscher selbst, für ihn sei „die Endphase der Weimarer Republik auch eine Zeit der vertanen Chancen, da sie in die Katastrophe des Dritten Reichs mündet - aber nicht zwangsläufig münden musste" ${ }^{10}$. In der Gegenüberstellung der gewählten

4. - Vgl. Moritz Föllmer, Rüdiger Graf (Hrsg.), Die „Krise“ der Weimarer Republik.Zur Kritik eines Deutungsmusters, Frankfurt/Main, New York, Campus, 2005.

5. - Reinhart Koselleck, „Krise“, in: Geschichtliche Grundbegriffe. Historisches Lexikon zur politisch-sozialen Sprache. Hrsg. von Otto Brunner, Werner Conze, Reinhart Koselleck, Bd. 3. Stuttgart, Klett, 1982, S. 617-650, hier S. 623.

6. - Ernst Bloch, „Philosophische Ansicht des Detektivromans“ [1960/1965], in: Jochen Vogt (Hrsg.), Der Kriminalroman: Poetik - Theorie - Geschichte, München, Fink, 1998, S. 38-51, hier S. 44.

7. - Robert Hültner, Die Godin [1997], München, btb, ${ }^{41999, ~ S . ~} 244$.

8. - Bisher deckt die Serie mit Der nasse Fisch (DnF), Der stumme Tod (DsT), Goldstein (G), Die Akte Vaterland (DAV) und Märzgefallene (M) die Jahre 1929 bis $1933 \mathrm{ab}$. S. 34.

9. - Hardy Reich, Die Machenschaften des Dr. Schmincke, in: FAZ, 01.02.2008,

10. - Interview mit Volker Kutscher, mobil, September 2007. Zit. n. http://www. gereonrath.de/der-autor.html. 
Formulierungen deutet sich eine Spannung an zwischen dem Wissen um die (bekannte) katastrophische Verlaufsform und der geplanten Verfertigung einer (offen gehaltenen) Krisengeschichte. Kutscher will nach eigenem Bekunden im realistischen Repräsentationsmodus vom krisenhaften wie schillernden Möglichkeitsraum der Weimarer Republik erzählen, stellt seine (Para-)Texte aber so zurecht, dass man in der ,schulfunkkorrekt[en]"11 Aufbereitung der Vergangenheit eben nicht nur die Kippfigur der Krise erkennt, sondern den Weg in den Untergang. In diesem Sinn lässt sich Thomas Wörtches böse Charakterisierung „Guido Knopp goes crime"12 für eine Analyse des Romanprojektes fruchtbar machen: Vor der historisch schlüssigen Kulisse der Jahre 1929 bis 1933 wird jenes geschichtete Konglomerat an Vorstellungen, Ereignissen und geschichtlichen Konstellationen zur Aufführung gebracht, auf das sich ein deutschsprachiges Publikum in seiner kollektiven Erinnerung an und in seinem Wissen um das Ende der Weimarer Republik verständigt hat. Im Bemühen, in der vermeintlich ungedeuteten Reproduktion damaliger Krisentopoi mimetisch an die Wahrnehmung der Zeitgenossen anzuschließen, türmt die Romanserie dabei Krisendiagnose auf Krisendiagnose. Der sich bei Denis Scheck einstellende Eindruck ,plumpe[r] Besserwisser-Prosa“13 ist erzählstrategischer Effekt einer Romanreihe, die eigentlich nicht sagen will, was sie weiß, aber in makrostruktureller Ordnung, Figurencharakterisierung und dem seismographischen Verweisungszusammenhang zwischen den behandelten Kriminalfällen und ihrem historischen Kontext einen Bedeutungsüberschuss produziert, mit dem sie für ihre Leser überdeutlich zur Schau stellt, dass sie das Ende kennt. Den Möglichkeitsraum der Weimarer Republik reduziert die Serie dabei - betrachtet man die zentrale Figur Gereon Rath - auf einen resignativen Normalisierungsdiskurs ${ }^{14}$.

Makrostrukturell knüpft Kutschers Romanserie an die historische Arbeit am Mythos der Goldenen Zwanziger Jahre an: Wir begegnen Gereon Rath erstmals im Jahr 1929 in Berlin, wo er, so informiert uns der Klappentext, ,eine Stadt im Rausch [erlebt]“. Der Roman formiert diese im Paratext verankerte Deutung einer orientierungslosen und radikalisierten Gesellschaft, die ihre einstigen Ordnungsmodelle

11. - Thomas Wörtche, Crime Watch Nr. 124, in: Der Freitag, 31.08.2007, online verfügbar unter https://www.freitag.de/autoren/der-freitag/crime-watch-no-124.

12. - Ebd.

13. - Denis Scheck in Druckfrisch, Sendung vom 14.12.2014, online verfügbar unter http://www.daserste.de/information/wissen-kultur/druckfrisch/videos/die-top-tenbelletristik-118.html.

14. - Vgl. Jürgen Link, ,Zum Anteil apokalyptischer Szenerien an der Normalisierung der Krise“, in: Uta Fenske, Walburga Hülk, Gregor Schuhen (Hrsg.), Die Krise als Erzählung. Transdisziplinäre Perspektiven auf ein Narrativ der Moderne, Bielefeld, Transcript, 2013, S. 33-47. 
verloren hat, sogleich zu einem ersten Krisenszenario - wenn auch mit augenfällig komischem Zungenschlag: Mit den pornographischen Bildern des „Schmutzfink[en]“ (DnF, 20) König, ,auf denen Doppelgänger der Hohenzollern und anderer preußischer Prominenz in eindeutigen Positionen abgelichtet waren“ (DnF, 17), wird jenes engmaschige Netz an restriktiven Verhaltenskodices und präskriptiven Wertvorstellungen, für das die nationalen Symbolfiguren des Wilhelminischen Kaiserreiches einstehen, als Anachronismus enttarnt und mit der hedonistischen Gegenwart der späten 1920er Jahre überblendet, die in ihrem „Tanz auf dem Vulkan“15 keinen sittlich-moralischen und politischen Kompass mehr kennt. Die auf Rath fokalisierte Erzählerrede nimmt diese radikale Vermarktung sexueller Freizügigkeit und ehemaliger „Majestätsbeleidigung“ (DnF, 15) allerdings nur resignativ als Ausdruck des Allbekannten zur Kenntnis: „So war die Welt nun einmal, seit sie aus den Fugen geraten war. 1919 hatte die Revolution alle moralischen Werte auf den Kopf gestellt, 1923 die Inflation alle materiellen“ (DnF 22). Angesichts dieser paradigmatisch formulierten Wahrnehmung eines Wertevakuums in Folge des Umsturzes scheint es der Frage wert, wie die Romane generell mit jenen Ereignissen und Krisenentwicklungen umgehen, die auf makrostruktureller Ebene zu den narrativen Knotenpunkten der historiographischen Fallerzählung zur Weimarer Republik gezählt werden. Zu diesen in den Romanen aufgegriffenen ,großen Ereignissen gehören u.a. die Wirtschafskrise samt ihrer sozialpsychologischen Auswirkungen, der Übergang zu den Präsidialkabinetten 1930, die Bankenkrise 1931, die Reichsexekution gegen Preußen 1932, die Ernennung Adolf Hitlers zum Reichskanzler 1933, der Reichstagsbrand sowie das daran geknüpfte ,Ermächtigungsgesetz: In all diesen historischen Daten verdichten sich systemische Rahmenbedingungen zu symptomatischen Ereignissen der Desintegration und Auflösung des parlamentarischen Systems. Vor dem Hintergrund einer zunehmenden Politisierung des öffentlichen Raumes heben sie sich als Zäsuren ab, sind allerdings nicht zu trennen von längerfristigen kritischen Entwicklungsprozessen und Herausforderungslagen, die sich u.a. mit den Schlagworten Antisemitismus und Erstarken extremer Ideologien, Angst vor einem Bürgerkrieg und Antiparlamentarismus, Nationalismus und DolchstoßLegende sowie einer allgemeinen existenziellen Verunsicherung angesichts der Modernisierungsschübe des 20. Jahrhunderts bezeichnen lassen.

15. - Vgl. Thomas W. Kniesche, Stephen Brockmann (Hrsg.), Dancing on the Volcano. Essays on the Culture of the Weimar Republic, Columbia SC, Camden House, 1994. 
Die Romane rufen diese Wegmarken nicht nur durch entsprechende Notizen auf den stets gleich gebauten Klappentexten als Bestandteil erlebter Wirklichkeit auf, sondern bilden die Prozesse der politischen Radikalisierung und der beginnenden Auflösung aus der Perspektive der Zeitgenossen durch eine fortgesetzte Neukalibrierung von Normalität ab. Dafür steht im Wesentlichen Gereon Rath ein, der sich über weite Strecken weigert, aus der erlebten Normalität des alltäglichen Geschehens besondere Ereignisse als krisenhaften Wendepunkt herauszuschälen. So vermag der karriereorientierte und traumatisierte Kölner, der aufgrund seiner Biographie sich selbst und Anderen immer wieder vorsagt „Von Politik verstehe ich nichts“ (DnF, 132), keinen prinzipiellen Unterschied zwischen den jeweiligen Berufungen Karl Friedrich Zörgiebels (SPD), Albert Grzesinskis (SPD), Kurt Melchers (DVP) und Magnus von Levetzows (NSDAP) zum Polizeipräsidenten Berlins zu erkennen:

„Unser Polizeipräsident ist Nazi, na und? Die sitzen ja jetzt auch in der Regierung. Als die Sozis noch regierten, waren die Polizeipräsidenten Sozis. Ob jemand ein guter Polizist ist, das zählte doch auf diesem Posten noch nie, da kam es immer nur auf das Parteibuch an“. (M, 177)

Während das historiographische Makronarrativ der Krise auf spezifische Zäsuren als Endpunkte einer noch entscheidungsoffenen Situation zuläuft und in der Konsequenz die Krisis als unausweichliches Moment der Entscheidung stets mitdenkt, wird dieses Strukturmuster in der Fokalisierung auf Rath ausgehebelt, denn für ihn ist dieses Symptom einer Politisierung des Polizeiapparates zwar bedauerlich, aber normal (M, 421). Diese ausgesagte Wahrnehmung von Normalität bzw. das Verharren in einer aussitzbaren „Latenzperiode“ 16 wird jedoch immer wieder unterlaufen und etwa in wiederholten Streitgesprächen zwischen den Figuren als erbittert verteidigte Selbstbeschwörungsformel und als Ausdruck verunsicherter Männlichkeit fassbar.

In der Romanserie ist dieses Muster, latente Herausforderungslagen als Teil des erlebten Alltags mitlaufen zu lassen und in den behandelten Kriminalfällen sowie herausgehobenen Momenten der Zeugenschaft zur schockhaften Erfahrung zu verdichten, wiederholt $\mathrm{zu}$ beobachten. Als Lackmus-Test für diese Überlegung lässt sich die literarische Repräsentation der Maiunruhen 1929 samt ihrer politischen Hintergründe anführen. Rath erlebt die Zuspitzung dieser blutigen

16. - Ansgar Nünning, ,Krise als Erzählung und Metapher: Literaturwissenschaftliche Bausteine für eine Metaphorologie und Narratologie von Krisen“, in: Carla Meyer, Katja Patzel-Mattern, Gerrit Jasper Schenk (Hrsg.), Krisengeschichte(n): „Krise“ als Leitbegriff und Erzählmuster in kulturwissenschaftlicher Perspektive, Stuttgart, Franz Steiner, 2013, S. 117-144, hier S. 124. 
Auseinandersetzungen im Zeichen des Antikommunismus, in denen sich die innenpolitische Spannung zwischen Polizei und Arbeitern entladen, in Der nasse Fisch hautnah mit - und kehrt sodann in eine „unwirklich“ scheinende Normalität zurück: „Alles wirkte wieder normal. Und die Normalität gleichzeitig unwirklich. Kaum zu glauben, dass wenige Kilometer entfernt der Ausnahmezustand herrschte, dass geschossen wurde, dass Menschen starben.“ (DnF, 79) Hier blitzt punktuell die zeitgenössische Normalität als zum Einsturz gebrachte, imaginäre Konstruktion auf, hinter der sich der Ausnahmezustand allenfalls noch in topographischer Einhegung verbergen lässt. Während auf historiographischer Ebene das durch den Krisenbeobachter Rath geschilderte Erlebnis des ,Blutmais“ insofern einen entwicklungsgeschichtlichen Wendepunkt darstellt, als es zum einen die Betriebsblindheit der Weimarer Republik auf dem rechten Auge exemplarisch verdeutlicht und zum anderen der KPD ,den eklatanten Beweis für die ,sozialfaschistische" Rolle der SPD-Führer"17 lieferte und somit mitverantwortlich zeichnet für die Spaltung der Arbeiterschaft, erlaubt uns der Text in der Fokalisierung auf Rath mitzuerleben, wie diese Denormalisierungserfahrung nach und nach in den Hintergrund gedrängt wird. An die Stelle der großen Frage nach der Wahrheit und ihrer narrativen Verfertigung (DnF, 103) tritt die reflexive Anpassung an die Verhältnisse, um ,,in diesem Spiel mitzuspielen“ (DnF, 107). In der mimetischen Simulation einer zeitgenössischen Froschperspektive stecken die Texte so die Bereiche des Wiss- und Deutbaren ab und sichern über die kalkulierte Verteilung von Augenzeugenschaft zugleich die Plausibilität widerstreitender Positionen. Während Charlotte Ritter in Die Akte Vaterland mit dem sog. Preußenschlag 1932 das „Ende“ der ,preußische[n] Demokratie“ (DAV, 420), also eines jener ,großen Ereignisse" der Weimarer Zeit erlebt, wirkt das Aufmarschieren der SA in einer masurischen Kleinstadt auf Gereon Rath „,beinahe idyllisch. Als würde es irgendwie dazugehören: die SA auf dem Weg zum Mittagessen, auch sie ein Teil des kleinstädtischen Lebens.“ (DAV, 196) ${ }^{18}$ Dieser Normalisierungsdiagnose eines bereits erfolgten Einzuges des Nationalsozialismus in den bürgerlichen Alltag - zumindest an der Peripherie - kontrastiert in Goldstein die Fassungslosigkeit Raths, als er die schmale Zone großstädtischer Normalität von der Brutalität der SA-Truppen aufgezehrt sieht: „Für Rath war es ein Schock, diese elegante, bürgerliche Gegend, für ihn immer ein Hort der Normalität in der wahnsinnigen Stadt, als Schauplatz solch massiver Straßenkrawalle

17. - Ursula Büttner, Weimar. Die überforderte Republik 1918-1933, Bonn, Bundeszentrale für politische Bildung, 2008, S. 396.

18. - Dieses Muster wiederholt sich in Märzgefallene: Während Charlotte den brennenden Reichstag beobachtet, amüsiert sich Gereon auf dem Kölner Karneval. 
zu erleben.“ (G, 571) Die Eindringlichkeit dieses Schreckens löst sich jedoch schnell auf und von der konkreten Bedrohung durch rechts ab: Obgleich der Roman in der Schilderung jener pogromartigen Gewalttaten für den heutigen Leser deutlich macht, dass der im Alltag als so normal wie konsensfähig gezeichnete Antisemitismus nicht über Nacht zur Staatsräson wird, sondern bereits zuvor jenes vom Staat in der Krise eröffnete Machtvakuum besetzt, entlässt er Rath doch am Ende in die Normalität der Kneipe: „Die Ereignisse draußen auf der Straße wirkten schon jetzt so unwirklich wie ein schlechter Traum.“ (G, 574) Ebenso wie in Der nasse Fisch kehren wir also von der Unwirklichkeit in die Wirklichkeit zurück, vom Ausnahmezustand in eine kritische Ordnung, in der Rath trotz des erfahrenen Bruches immer noch darauf beharren kann, ,für Recht und Ordnung und für sichere Straßen“ (DnF, 173) zu sorgen.

In den Streitgesprächen mit Charlotte Ritter erscheint dabei die je gegensätzliche Bewertung der vorliegenden Situation als Spannung zwischen einer kurzsichtigen Evaluierung, die sich allein am Maßstab persönlicher Betroffenheit orientiert, und einem Standpunkt, der in der Ausrichtung auf die Zukunft eher dem Bewertungshorizont heutiger Leser entspricht:

„Ich kann es nur nicht ertragen, dass Achim von Roddeck den begnadeten Schriftsteller und aufrechten Deutschen spielt, der perfekt in die neue Zeit passt, während er eigentlich ein Arschloch und Lügner und Mörder ist.“

„Vielleicht passt er gerade deswegen so gut in die neue Zeit.“

„Du musst nicht immer gleich politisch werden.“

„Ich werde nicht politisch, das Leben ist politisch. Unsere ganze Arbeit ist politisch.“

„Meine nicht. Mir geht es nur darum, Mörder nicht ungeschoren davonkommen zu lassen.“ (M, 470)

Während Charlotte im Zitat der nationalsozialistischen Redeformel von der neuen Zeit auf ein abgeschlossenes vorher verweist und damit im historiographischen Sinne einer Zäsur das Wort redet, weigert sich Gereon über den vorliegenden Fall hinaus zu denken, schließlich werde sich - so schlussfolgert er einen Tag nach Verabschiedung des Ermächtigungsgesetzes - die politische Lage nach den ersten „Auswüchse[n]“ sicherlich „wieder normalisieren“ (M, 389). Vertreten wird so ein Konzept des unentschiedenen Ausharrens in einer als kontingent und chaotisch erlebten Gegenwart, die aufgrund ihrer systemischen Fehlentwicklungen nicht $\mathrm{zu}$ ändern ist, und des Rückzugs auf jenen eigenen Handlungsraum, den Gereon als Kern seines Selbstverständnisses so listenreich wie manipulativ verteidigt: seine Position als Kriminalkommissar der Inspektion A. Die damit einher- 
gehende Modulation von einer apolitischen Haltung in Opportunismus, die die Weichen zu stellen sucht für die weiteren Romane, trifft sich mit der narrativen Entfaltung eines Profils, das sich nur als Krisenknäuel bezeichnen lässt. Ausgestattet mit hard boiled-Topoi, die Rath als Maske immer wieder an- und ausprobiert, und einer an Henning Mankell geschulten Gequältheit der Ermittlerfigur durch ihre inneren „Dämonen“ (DsT, 71) zeichnet sich die psychosoziale Blaupause einer Männlichkeit in der Krise ab. Eingeführt als traumatisierter Charakter, der in seinem Karrierestreben um Autonomie, Handlungsfähigkeit und Individualität ringt, sehen wir ihn in Der stumme Tod als strafversetzten lone hero mit Liebeskummer, auf den die Presse, die Unterwelt, die Polizei und der Vater erheblichen Druck ausüben - eine Konstellation, die mit kleinen Abweichungen alle Romane prägt. Die nach außen kommunizierte apolitische Haltung erscheint dabei wesentlich als Widerstand gegen die übermächtige Autorität des Vaters und dessen kölsche Klüngelei, gegen die Rath sein Berufscredo positioniert: „Politik macht die Straße zu einem Schlachtfeld.“ (DsT, 367) Für Rath ist die Arbeit als Polizeibeamter die einzig verlässliche Konstante seines Selbstverständnisses, aber selbst dieses Refugium sieht sich von Sinnlosigkeit umstellt - sei es in der Inspektion E (DnF, 23), in der Mordkommission (DAV, 25) oder bei der Politischen Polizei (M, 539).

In das Leben dieser gespaltenen Figur lassen die Texte als historische Kriminalromane routiniert heteronome Ereignisse einbrechen, die in ihrer krisenhaften Denormalisierung erhebliche, wenn auch routinierte Handlungsimpulse freisetzen: Ausgehend vom Fund einer Leiche verfolgen die Aufklärungsgeschichten das An- und Ablaufen des polizeilichen Ermittlungsapparates samt Mordauto, Mittagessen bei Aschinger und männerbündischen Revierkämpfen. Auffällig ist, dass die für den Leser stets eindeutig in ihren weitreichenden innenpolitischen Verästelungen aufgeklärten Mordfälle in ihrer Serialität ein zentrales Krisensymptom eskalieren lassen: die zunächst latente und allererst aufzudeckende, dann offen zu Tage tretende Politisierung und Kriminalisierung des Polizeiapparats. In den diversen Verschwörungsplots - sei es der Waffenschmuggel in Der nasse Fisch oder der ,geheime Zusammenschluss frustrierter Polizisten“ in Goldstein, „die sich entschlossen hatten, [...] auch noch Richter und Henker in Personalunion zu spielen“ (G, 545) - lässt sich so einerseits das Abgleiten der Polizei in die Anomalie beobachten. Andererseits schaffen die Texte mit den behandelten Fällen einen deutlichen Verweisungszusammenhang zwischen (fiktionalem) Ereignis und Struktur und produzieren in der Konsequenz einen (historiographischen) Bedeutungsüberschuss, der allein vom Leser aufzuschlüsseln ist. Situiert im Vorfeld der Staatskrise 1930 erzählt etwa Der stumme Tod vom Untergang des Stummfilmes und wählt dazu einen Serienmörderplot, 
in dem der Täter über die zeichenhafte Überhöhung der Tatorte und die symbolische Zurichtung seiner Opfer seine Geschichte erzählt - und lässt im Hintergrund den Fall Horst Wessel mitlaufen, in dem sich mit der propagandistischen Märtyrerkonfiguration eine andere Spielart des Zusammenschlusses von totem Körper und politischer Botschaft zeigt. Diese Kommunikation mit dem Leser setzt am deutlichsten der Titel jenes Rath-Bandes um, der auf historiographischer Ebene die Entscheidung der Weimarer Krise vertritt: Märzgefallene. Denn während dieser Begriff von den Nationalsozialisten besetzt ist, die damit all jene ,nach den Reichstagswahlen am 5. März 1933 in die NSDAP eingetretenen Opportunisten" 19 bezeichnen, firmiert er im Roman als Titel eines autobiographischen Manuskripts um den Ersten Weltkrieg, mit dem Achim Freiherr von Roddeck eigentlich den untergetauchten Benjamin Engel aus der Reserve locken möchte, zugleich aber das Wohlwollen der neuen Machthaber auf sich zieht: „Der böse, geheimnisvolle Jude, der umherzieht wie Ahasver und brave deutsche Veteranen abschlachtet. Das hört sich doch an wie der Fortsetzungsroman aus dem Stürmer.“ (M, 269)

Volker Kutschers Krimiserie, so lässt sich zusammenfassend festhalten, arbeitet sich ab an der Krise als Metapher und Narrativ und wählt dazu gezielt einen apolitischen Krisenbeobachter, der sich im Rahmen seines beruflich vorgezeichneten Normalisierungsdiskurses und - so formuliert es Charlotte Ritter - in einer ,ungesunden Mischung aus Arroganz und Ignoranz" (M, 178) einer Strategie des „Weiterwurstelns und Aussitzens" 20 verschreibt. Die eingangs zitierte Idee Kutschers, den Möglichkeitsraum Weimar literarisch einzufangen, setzen die Romane folglich um, indem sie die Ereignishaftigkeit spezifischer historischer Daten sowohl auszehren als auch akzentuieren und dabei beobachten, wie die Denormalisierungserfahrungen in eine ungebrochene Hoffnung auf Stabilität und Kontinuität in den Grenzen einer jeweils neu aufgerichteten Normalität übersetzt werden. Die Serie bemüht sich mithin am Beispiel Raths plausibel und nachvollziehbar zu erzählen, wie man trotz Kenntnis der ,insider story' den entwicklungsgeschichtlichen Wendepunkt verpassen kann. Der über Rath erzählte Normalisierungsdiskurs geht so nicht in politischer Naivität bzw. Dummheit auf, sondern verweist vielmehr auf eine beeindrukkende Flexibilität in der Verfolgung der eigenen Karriere. Aus erinnerungspolitischer Warte ist diese Erzählstrategie allerdings bedenklich: Für die intradiegetische Simulation einer zeitgenössischen Perspektive zerbrechen die Romane die historiographische Krisenerzählung sowie die an sie geknüpften Erklärungsmuster für das Scheitern der Weimarer

19. - Cornelia Schmitz-Berning, Vokabular des Nationalsozialismus, Berlin, New York, de Gruyter, 2007, S. 399.

20. - Nünning, Krise als Erzählung und Metapher, a.a.O., S. 134. 
Republik und geben sie in weiträumiger Streuung als hypothetische Wissensfragmente ihren Figuren zurück, so dass für den Leser nur eine überdeterminierte Krisenlage fassbar wird. Die immer wieder aufs Neue vorgeführte Infragestellung und Aushandlung von Normalität vertritt dabei notdürftig die eingangs angekündigte Auslotung „,vertane[r] Chancen" 21 , bedient aber dabei im Wissen um die Katastrophe im Wesentlichen zwei Narrative, die auf den Nationalsozialismus zulaufen. Denn zum einen scheint die Serie uns mit Raths Normalisierungswillen auf jene ganz normalen Männer vorzubereiten, denen wir in Christopher Brownings gleichnamiger Studie begegnen und denen das Morden zum „Alltag“22 geworden ist. Aus diesem Blickwinkel lässt sich die hier vorgelegte Normalisierung der Weimarer Republik nicht trennen von der Normalisierung des ,Dritten Reiches ${ }^{623}$. Zum anderen konturiert sich durch die Rückbindung von zeitdiagnostischem Erkennen an Augenzeugenschaft die nach 1945 berühmte Aussageformel des NichtWissens, während politische Wachsamkeit als Symptom hysterischer Weiblichkeit firmiert. Märzgefallene verabschiedet den ,mittleren Helden' denn auch mit der beunruhigenden Ahnung, dass die Krise längst entschieden ist und eine neue Normalität bereits Einzug gehalten hat: „In einer Stadt ohne Hakenkreuze. Erst in Paris war Rath klargeworden, wie sehr Berlin sich in wenigen Wochen verändert hatte." $(\mathrm{M}, 600)$.

21. - Interview mit Kutscher, 2007, a.a.O.

22. - Christopher Browning, Ganz normale Männer. Das Reserve-Polizeibataillon 101 und die „Endlösung“ in Polen [1992], Reinbek bei Hamburg, Rowohlt, 1993, S. 16.

23. - Vgl. Gavriel D. Rosenfeld, Hi Hitler! How the Nazi Past is Being Normalized in Contemporary Culture, Cambridge, Cambridge University Press, 2015. 